\title{
Analytic Properties of the Quaternion Function
}

\author{
Youcai Xue \\ School of Science, Zhejiang University of Science \& Technology, Zhejiang 310023, China
}

\begin{abstract}
Many properties of complex functions are pretty difficult to be generalized in the field of quaternion function, as the commutative law of multiplication fails in the latter. The derivative of quaternion function is defined in this paper. Moreover, by the similar method in judging the analytic property of complex function, Cauchy-Riemann equation is used to determine the analytic property of quaternion function. Furthermore, several concrete examples are discussed, and certain errors in (P. W. Yang. 2009) are pointed out as well.
\end{abstract}

Keywords: Quaternion function, Analytic function, Cauchy-Riemann equation, Derivative formula

\section{Introduction}

The quaternion function is an important aspect of functions theory. With a similar method of Cauchy-Riemann equation in complex functions, (P. W. Yang. 2009) defines the analytic of quaternion function directly. However, the definition may lead to some mistakes. We define the derivative of quaternion function and give a different definition of its analytic property. Moreover, we also present the derivative formulas as well as some examples.

Throughout the whole paper, let $\mathrm{R}$ and $\mathrm{C}$ denote the fields of real numbers and complex numbers respectively. Set $Q=\left\{q=x_{1}+x_{2} i+x_{3} j+x_{4} k \mid x_{1}, x_{2}, x_{3}, x_{4} \in R\right\}$ be a real quaternion field. Assume $D$ is a region in $R^{4}$ and $f(q)$ is a quaternion function defined in $D$. Let $x=\left\{x_{1}, x_{2}, x_{3}, x_{4}\right\}$ be some element in $R^{4}$, such that $U: D \rightarrow Q ; w=f(q)=u_{1}+u_{2} i+u_{3} j+u_{4} k$, where $u_{i}\left(x_{1}, x_{2}, x_{3}, x_{4}\right), i=1,2,3,4$ are real functions defined on $D$.

Definition 1.1. Let $w=f(q)$ be the quaternion function defined in the certain neighborhood of $q_{0}$, or some region $D$ containing $q_{0}$, we could consider the quotient

$$
\frac{\Delta w}{\Delta q}=\frac{f(q)-f\left(q_{0}\right)}{q-q_{0}}=\frac{f\left(q_{0}+\Delta q\right)-f\left(q_{0}\right)}{\Delta q}, \Delta q \neq 0
$$

If the limit of quotient exists whenever a point $q$ approaches to the point $q_{0}$ arbitrarily, or equivalently speaking, the difference $\Delta q$ vanishes in any arbitrary direction, the limit is called the derivative of the function $f(q)$ at a given point $q_{0}$, and denoted by $f^{\prime}\left(q_{0}\right)$ or $\frac{d w}{d q}$,

$$
f^{\prime}\left(q_{0}\right)=\lim _{\Delta q \rightarrow 0} \frac{\Delta w}{\Delta q}=\lim _{q \rightarrow q_{0}} \frac{f(q)-f\left(q_{0}\right)}{q-q_{0}}
$$

Let the function $f(q)$ is derivative at a point $q_{0}$, then $\lim _{\Delta q \rightarrow 0} \frac{\Delta w}{\Delta q}=f^{\prime}(q)$ holds, i.e.

$\frac{\Delta w}{\Delta q}=f^{\prime}(q)+\eta, \lim _{\Delta q \rightarrow 0} \eta=0$. Hence, $\Delta w=f^{\prime}(q) \Delta q+\varepsilon$, where the remainder $|\varepsilon|=|\eta \cdot \Delta q|$ is a higher order infinitesimal of the difference $|\Delta q|$.

Definition 1.2. Define $f^{\prime}(q) \Delta q$ is the difference of $f(q)$, and denote to be $d w$ or $d f(q)$, i.e.

$$
d w=f^{\prime}(q) \Delta q
$$

Taking $f(q)=q$ specifically, $d q=\Delta q$ holds. Then $d w=f^{\prime}(q) d q$,(i.e.) $\frac{d w}{d q}=f^{\prime}(q)$ follows by (1.2). Therefore, this implies the equivalence between differentiability and derivative of some certain function $f(q)$. 
Example1.1. Let the function $f(q)=q^{n}$ ( $n$ should be integers) be differentiable everywhere in $R^{4}$, so that

$$
f^{\prime}(q)=n q^{n-1}
$$

Example1.2. Let the function $f(q)=\bar{q}=x_{1}-x_{2} i-x_{3} j-x_{4} k$, then it isn't differentiable anywhere in $R^{4}$.

It's clear that the differentiability of the function $f(q)$ at the given point $q$ should imply its continuity. However, the converse may not hold according to the second example. Speaking more precisely, to a given point $q$, the continuity of the function could not deduce the differentiability at that point.

Let two functions $f_{1}(q), f_{2}(q)$ both be differentiable at the point $q$, one can easily verify that

$$
\begin{aligned}
& \left.\left[f_{1}(q) \pm f_{2}(q)\right]\right)=f_{1}^{\prime}(q) \pm f_{2}^{\prime}(q) \\
& {\left[f_{1}(q) f_{2}(q)\right]^{\prime}=f_{1}^{\prime}(q) f_{2}(q)+f_{1}(q) f_{2}^{\prime}(q)} \\
& {\left[\frac{f_{1}(q)}{f_{2}(q)}\right]^{\prime}=\frac{f_{1}^{\prime}(q) f_{2}(q)-f_{1}(q) f_{2}^{\prime}(q)}{f_{2}^{2}(q)}, f_{2}(q) \neq 0}
\end{aligned}
$$

2. Main Results

Definition2.1 The quaternion function $f(q)$ is an analytical function in $D$, if $f(q)=u_{1}+u_{2} i+u_{3} j+u_{4} k$ is defined properly in $D\left(\subset R^{4}\right), u_{i}\left(x_{1}, x_{2}, x_{3}, x_{4}\right)$ are differentiable in this region, and the following holds

$$
\left\{\begin{array}{l}
\frac{\partial u_{1}}{\partial x_{1}}-\frac{\partial u_{2}}{\partial x_{2}}+\frac{\partial u_{3}}{\partial x_{3}}-\frac{\partial u_{4}}{\partial x_{4}}=0 \\
\frac{\partial u_{1}}{\partial x_{2}}+\frac{\partial u_{2}}{\partial x_{1}}-\frac{\partial u_{3}}{\partial x_{4}}-\frac{\partial u_{4}}{\partial x_{3}}=0 \\
\frac{\partial u_{1}}{\partial x_{3}}-\frac{\partial u_{2}}{\partial x_{4}}+\frac{\partial u_{3}}{\partial x_{1}}-\frac{\partial u_{4}}{\partial x_{2}}=0 \\
\frac{\partial u_{1}}{\partial x_{4}}-\frac{\partial u_{2}}{\partial x_{3}}-\frac{\partial u_{3}}{\partial x_{2}}+\frac{\partial u_{4}}{\partial x_{1}}=0
\end{array} \quad\left(\begin{array}{ccccc}
\frac{\partial}{\partial x_{1}} & -\frac{\partial}{\partial x_{2}} & \frac{\partial}{\partial x_{3}} & -\frac{\partial}{\partial x_{4}} \\
\frac{\partial}{\partial x_{2}} & \frac{\partial}{\partial x_{1}} & -\frac{\partial}{\partial x_{4}} & -\frac{\partial}{\partial x_{3}} \\
\frac{\partial}{\partial x_{3}} & -\frac{\partial}{\partial x_{4}} & \frac{\partial}{\partial x_{1}} & -\frac{\partial}{\partial x_{2}} \\
\frac{\partial}{\partial x_{4}} & -\frac{\partial}{\partial x_{3}} & -\frac{\partial}{\partial x_{2}} & \frac{\partial}{\partial x_{1}}
\end{array}\right)\left(\begin{array}{l}
u_{1} \\
u_{2} \\
u_{3} \\
u_{4}
\end{array}\right)=O\right.
$$

The previous system of partial differential equations could appropriately be viewed as a generalized version of the famous Cauchy-Riemann equation in complex functions.

Theorem2.1. Let the function $f(q)=u_{1}+u_{2} i+u_{3} j+u_{4} k$ be properly defined and analytic in region $D\left(\subset R^{4}\right)$, then

$$
\begin{aligned}
f^{\prime}(q) & =\frac{\partial u_{1}}{\partial x_{1}}+i \frac{\partial u_{2}}{\partial x_{1}}+j \frac{\partial u_{3}}{\partial x_{1}}+k \frac{\partial u_{4}}{\partial x_{1}}=\frac{\partial u_{2}}{\partial x_{2}}-i \frac{\partial u_{1}}{\partial x_{2}}+j \frac{\partial u_{4}}{\partial x_{2}}-k \frac{\partial u_{3}}{\partial x_{2}} \\
& =\frac{\partial u_{3}}{\partial x_{3}}-i \frac{\partial u_{4}}{\partial x_{3}}-j \frac{\partial u_{1}}{\partial x_{3}}+k \frac{\partial u_{2}}{\partial x_{3}}=\frac{\partial u_{4}}{\partial x_{4}}+i \frac{\partial u_{3}}{\partial x_{4}}-j \frac{\partial u_{2}}{\partial x_{4}}-k \frac{\partial u_{1}}{\partial x_{4}}
\end{aligned}
$$

Proof. Since $f(q)=u_{1}+u_{2} i+u_{3} j+u_{4} k$ is analytic in region $D$, and $q \in D$, then $f(q)$ is differentiable at same point $q$, and the following limit holds

$$
\lim _{\Delta q \rightarrow 0} \frac{f(q+\Delta q)-f(q)}{\Delta q}=f^{\prime}(q)
$$

Let the differences of quaternion functions be

$\Delta q=\Delta x_{1}+i \Delta x_{2}+j \Delta x_{3}+k \Delta x_{4}, \quad f(q+\Delta q)-f(q)=\Delta u_{1}+i \Delta u_{2}+j \Delta u_{3}+k \Delta u_{4}$,

and denote $\Delta u_{i}=u_{i}\left(x_{1}+\Delta x_{1}, x_{2}+\Delta x_{2}, x_{3}+\Delta x_{3}, x_{4}+\Delta x_{4}\right)-u_{i}\left(x_{1}, x_{2}, x_{3}, x_{4}\right)$, then we could rewrite down the form of the limit(2.3)in a concise way 


$$
\lim _{\substack{\Delta x_{m} \rightarrow 0 \\ m=1,2,3,4}} \frac{\Delta u_{1}+i \Delta u_{2}+j \Delta u_{3}+k \Delta u_{4}}{\Delta x_{1}+i \Delta x_{2}+j \Delta x_{3}+k \Delta x_{4}}=f^{\prime}(q)
$$

The limit(2.3)always exists whenever the difference of quaternion functions $\Delta q$ vanishes in any arbitrary direction. With the preceding assumption, we could set $\Delta x_{1} \rightarrow 0$, and $\Delta x_{2}=\Delta x_{3}=\Delta x_{4}=0$. Then we could derive another elegant formula from (2.4)and(2.3)

$$
\lim _{\Delta x_{1} \rightarrow 0}\left(\frac{\Delta u_{1}}{\Delta x_{1}}+i \frac{\Delta u_{2}}{\Delta x_{1}}+j \frac{\Delta u_{3}}{\Delta x_{1}}+k \frac{\Delta u_{4}}{\Delta x_{1}}\right)=f^{\prime}(q),
$$

Therefore, four partial derivatives $\frac{\partial u_{1}}{\partial x_{1}}, \frac{\partial u_{2}}{\partial x_{1}}, \frac{\partial u_{3}}{\partial x_{1}}, \frac{\partial u_{4}}{\partial x_{1}}$ all exist, and such that

$$
f^{\prime}(q)=\frac{\partial u_{1}}{\partial x_{1}}+i \frac{\partial u_{2}}{\partial x_{1}}+j \frac{\partial u_{3}}{\partial x_{1}}+k \frac{\partial u_{4}}{\partial x_{1}}
$$

Other formulas in(2.2)follows in a similar way.

Example 2.1 Discuss the analyticity of the function $f(q)=q^{2}=\left(x_{1}+i x_{2}+j x_{3}+k x_{4}\right)^{2}$.

Solution: The function $f(q)$ is well defined in entire $R^{4}$, and with some computations,

we could obtain that $q^{2}=\left(x_{1}^{2}-x_{2}^{2}-x_{3}^{2}-x_{4}^{2}\right)+2 x_{1} x_{2} i+2 x_{1} x_{3} j+2 x_{1} x_{4} k$.

By the definition(2.1), it's easy to verify this function is analytic in $R^{4}$, and such that

$$
\left(q^{2}\right)^{\prime}=\frac{\partial u_{1}}{\partial x_{1}}+i \frac{\partial u_{2}}{\partial x_{1}}+j \frac{\partial u_{3}}{\partial x_{1}}+k \frac{\partial u_{4}}{\partial x_{1}}=2 x_{1}+2 x_{2} i+2 x_{3} k+2 x_{4} k=2 q
$$

Therefore, this completes the claim that the function $q^{2}$ is analytic in $R^{4}$. By a similar method, we could prove that the function $f(q)=q^{n}$ is analytic in $R^{4}$ and $\left(q^{n}\right)^{\prime}=n q^{n-1}$.

Theorem 2.2 Let two functions $f_{1}(q), f_{2}(q)$ be analytic in region $D$, then $f_{1}(q) \pm f_{2}(q), \quad f_{1}(q) \cdot f_{2}(q), \quad \frac{f_{1}(q)}{f_{2}(q)},\left(f_{2}(q) \neq 0\right)$ are all analytic functions in the same region $D$.

Example2.2. Recalling Theorem 2.2 and Example2.1, the polynomial function $f(q)=a_{n} q^{n}+a_{n-1} q^{n-1}+\cdots+a_{1} q+a_{0}, a_{i} \in R(i=0,1, \cdots, n)$ is analytic in $R^{4}$.

Thus, the fractional polynomial function

$$
f(q)=\frac{f_{1}(q)}{f_{2}(q)}=\frac{a_{n} q^{n}+a_{n-1} q^{n-1}+\cdots+a_{1} q+a_{0}}{b_{m} q^{m}+b_{m-1} q^{m-1}+\cdots+b_{1} q+b_{0}}, a_{i}, b_{j} \in R(i=0,1, \cdots, n, j=1,2, \cdots, m)
$$

is analytic, where $f_{2}(q) \neq 0$.

By the way, the errors of definitions of analytic functions via two operators(4)and(8)are unavoidable in (P. W. Yang. 2009), because even a simple function $f(q)=q^{2}$ could not be valid by the definition described in it.

\section{Further open problems.}

In the successive articles, we would like to discuss the analyticity of some other elementary functions of a quaternion variable, i.e. exponential function and logarithmic function etc.

\section{References}

P. W. Yang. (2009). Mathematical Analysis of Quaternion numbers And Partial Differential Equation, (first edition), Scientific Press, (2009):4-6.

Y. Q. Zhong. (2003). Theory of The functions of a complex variable, (third edition), Higher Education Press, (2003):50-56. 